\title{
Charged null fluid and the weak energy condition
}

\author{
Amos Ori \\ Theoretical Astrophysics, California Institute of Technology, Pasadena, CA 91125, USA
}

Received 17 January 1991

\begin{abstract}
We use the Einstein-Maxwell field equations to derive the fundamental equation of motion for charged null fluid. This equation of motion includes a Lorentz force term. Using this equation, we show that charged null fluid always satisfies the weak energy condition. This result is in contrast to previous interpretations of the charged Vaidya solution (a special case of a charged null fluid), which produced violations of the weak energy condition. The errors in the previous interpretations are explained. The new interpretation is then applied explicitly to the charged Vaidya solution.
\end{abstract}

\section{Introduction}

The evolution of spherically symmetric electrically charged null fluid has been considered by several authors [1-3]. Charged null fluid may be regarded as a stream of non-interacting massless charged particles ('charged photons'), which interact only with the gravitational and electromagnetic fields. The exact solution of the MaxwellEinstein equations for spherical charged null fluid, the charged Vaidya solution [1], is a straightforward generalization of both the Reissner-Nordstrom and the Vaidya solutions. This solution is most easily expressed in $(r, v)$ coordinates, where $v$ is a comoving, ingoing null coordinate and $r$ is the area coordinate (see equation (2)). The solution depends on two arbitrary functions, $m(v)$ and $e(v)$, which express the total mass and electric charge (respectively) enclosed within the shell labelled $v$. The dependence of $m$ and $e$ on $v$ reflects the presence of the charged photons, which carries both energy and electric charge.

Despite its simple and straightforward physical meaning, it has been thought that the charged Vaidya solution suffers from a fundamental difficulty. According to the standard interpretation of this solution, it can easily violate the weak energy condition [2-4]; i.e. an observer who moves on a timelike geodesic may measure a negative energy density. This happens if, for some shell $v, \mathrm{~d}\left(e^{2}\right) / \mathrm{d} m$ is positive. The (locally measured) energy density of such a fluid shell becomes negative whenever its $r$ value becomes smaller than some critical value, $r_{c}(v)=e \mathrm{~d} e / \mathrm{d} m[2,3]$. Due to the resulting flux of ingoing negative energy, the area of a Reissner-Nordstrom black hole may decrease, and eventually the black hole may even disappear [2]. This process was used in [4] as a classical model for the geometry of evaporating charged black holes.

For an inflow situation, the null fluid comes from large $r$ values, so that initially all the shells have $r>r_{\mathrm{c}}(v)$. The central question is, therefore, whether or not the charged particles can penetrate into the problematic region $r<r_{\mathrm{c}}(v)$. This requires a careful analysis of the equation of motion (EOM) for massless charged particles. 
Previous analyses were restricted to the spherically symmetric case, in which the charged fluid flows along radial null orbits. Since every radial null orbit runs along a null geodesic, it was only natural to assume that massless charged particles move on null geodesics, and do not feel the Lorentz force [2,3]. To make things conceptually clear, consider the radial orbits of massless charged test particles in a fixed ReissnerNordstrom geometry. These would seem to be the relevant orbits, even when the fluid carries non-negligible charge and mass, because, due to the spherical symmetry, each of the ingoing photons merely 'feels' the total mass $m(v)$ and the total charge $e(v)$ enclosed in its spherical shell $v$, and is insensitive to the distribution of the charge and the mass. In the Reissner-Nordstrom geometry, radial null geodesics go all the way from infinity to $r=0$. Assuming that charged photons do not feel the Lorentz force, nothing can prevent the particles from penetrating into the problematic region $r<r_{\mathrm{s}}^{\dagger}$, where their energy becomes negative. It is rather disturbing that such a simple model-a stream of massless charged particles-seems to give rise to the undesired result of negative energies.

What is the resolution of this difficulty? The only factor that might prevent the charged photons from penetrating into $r<r_{c}$ is the Lorentz repulsion. But in what way can this radial force influence the motion of radially moving particles, if their zero mass forces them to stay on radial null orbits? The main goal of this paper is to resolve this problem.

The complete local description of the motion of a massless particle is given by its wavevector $k^{\alpha}$. This vector determines the direction of motion, and the energy of the particle. The measured energy $E_{o b}$ (subscript ob for observed) of the particle is proportional to $k^{\alpha}$,

$$
E_{\mathrm{ob}}=c u_{\mathrm{ob}}^{\alpha} k_{\alpha}
$$

where $u_{o b}^{\alpha}$ is the 4-velocity of the observer. The constant $c$ can be chosen arbitrarily for each orbit, but it îs to be hela constant along the orbits. The arbitrariness of $c$ just reflects the freedom to scale the magnitude of $k^{\alpha}$ for each null orbit. Once the initial magnitude of $k^{\alpha}$ is given, its evolution along the orbit is to be determined from the EOM. Consider now two massless particles, one neutral and the other charged, which move on the same radial orbit (or on two equivalent orbits) in a Reissner-Nordstrom spacetime. Assume also that initially (at, say, $r=r_{1}$ ) the two particles have the same $k^{\alpha}$ (the same energy). Due to the spherical symmetry, the radial Lorentz force (if it exists) cannot disperse the orbits from each other. But it may induce a difference in the magnitude of $k^{\alpha}$. A detector at $r=r_{2}$ will then detect different energies for the two particles! Thus, even in the strictly spherically symmetric case, the Lorentz force may have a physical significance. In order to better understand the evolution of charged null fluid, it is worthwhile to abandon spherical symmetry and construct the fundamental EOM for massless charged particles. This fundamental EOM must be covariant, consistent with the field equations and applicable for generic situation (i.e. generic geometry, electromagnetic field and initial 4-momentum). We shall first show that the geodesic equation does not satisfy these requirements: for a generic charged null flow, it is incompatible with the Maxwell-Einstein equations. Then, by a straightforward gen. eralization of the EOM for massive charged particles, we shall obtain a covariant EOM which does include a Lorentz force term. This EOM is consistent with the field equations and we regard it as the fundamental equation of motion for massless charged particles.

+ In this test-particle context, the term de/ $\mathrm{d} m$ that appears in the above definition of $r_{\varepsilon}$ is to be understood as the ratio (total energy)/(electric charge) associated with the test particle. 
Returning to spherical situations, there still remains a serious difficulty. In a radial motion, even with a Lorentz force, the orbits must be parallel to one of the two radial null geodesics (ingoing or outgoing). How can the Lorentz force prevent the particles from penetrating into the problematic region $r<r_{\mathrm{c}}$ ? The answer is rather delicate. Locally, the orbits must be everywhere tangent to one of the two radial null geodesics. However, this does not mean that the orbits are globally tangent to null geodesics: at $r=r_{\mathrm{c}}, k^{\alpha}$ vanishes, and the tangent direction is not uniquely defined there. As $k^{\alpha}$ vanishes, the orbit can switch to the outgoing null direction without any discontinuity in $k^{\alpha}$ itself. A close inspection of the EOM at $r=r_{\mathrm{c}}$ reveals that it has a critical point there, and just reads $0=0$. Mathematically, there are two possible ways to continue the orbit there: it can either continue on its original ingoing null direction, or switch to the outgoing one.

Which of the two possibilities is the correct one? Continuation along the original ingoing direction is the more smooth and elegant. However, physical considerations demand the second continuation. To show this, we introduce a small perturbation of the initial 4-momentum direction, so it is no longer strictly radial. This removes the critical point, allowing one to calculate the orbit without any ambiguity. For small perturbations, the orbits show a transition from the original ingoing (almost) radial null direction to an outgoing one, and the transition occurs at $r \approx r_{c}$. Moreover, in the limit of vanishing perturbations, one precisely obtains the radial solution, which switches at $r=r_{\mathrm{c}}$ from the ingoing to the outgoing radial null direction.

The transition from ingoing to outgoing direction can be explained intuitively as follows. As the charged particle moves on the ingoing direction, it loses energy due to the Lorentz force. At $r=r_{c}$, its energy has been reduced to zero $\left(k^{\alpha}=0\right)$. This is the point where the transition to the outgoing direction takes place. Then, the particle begins to drift along the outgoing geodesic; gaining energy due to the Lorentz force. Note that the transition occurs when $k^{\alpha}$ vanishes; hence, the 'jump' in the direction of $k^{\alpha}$ does not indicate a discontinuity in $k^{\alpha}$ itself. Moreover, at this moment the 4-momentum may formally take any intermediate direction without violating the zero-mass condition $k_{\alpha} k^{\alpha}=0$.

Due to this transition, the energy of the particles is always positive (except at $r=r_{c}$, where it vanishes). Returning back to the charged Vaidya problem (in which the massless particles are no longer regarded as test particles), we find that the fluid energy density is everywhere positive, namely, there is no violation of the weak energy condition.

The positivity of energy density is not restricted to the spherically symmetric case. The energy density is positive in general-provided that $k^{\alpha}$ is everywhere futuredirected (see section 4). By itself, future-directedness is plausible and, indeed, we assume that initially the 4-momentum is future-directed. The question remains, however, whether the EOM guarantees that $k^{\alpha}$ remains future-directed forever. Due to its null character, any transition from future-directed to past-directed $k^{\alpha}$ must occur at a vanishing point-a point where the 4-momentum entirely vanishes. Mathematically, at the vanishing point the orbits may either continue along the same null direction (resulting in a negative energy density), or switch to another null direction for which the energy density remains positive. However, by studying the effect of perturbations on orbits that approach vanishing points, we shall show that the second possibility is always the correct one. We conclude, therefore, that charged null fluid always satisfies the weak energy condition.

The paper is organized as follows. In section 2 we shall briefly review the charged Vaidya solution and its standard interpretation, which results in a violation of the 
weak energy condition. In section 3 we shall use the field equations to derive the general EOM for massless charged particles. The interpretation of null fuid in terms of a conserved current of massless particles will also be given. In section 4 we shall discuss, for a generic model of charged null fluid, the question of positivity of the energy density. The related issue of vanishing points will also be discussed. In section 5 we shall analyse the orbits of massless charged particles in the Reissner-Nordstrom geometry, focusing attention on the behaviour near the vanishing points. We shall show how the orbits switch to the outgoing radial null direction.

In section 6 we shall use the framework of constant electromagnetic field in flat spacetime to analyse the behaviour of orbits near generic vanishing points. We shall show that, as in the spherically symmetric case, at a vanishing point there is a transition from one null eigendirection of the electromagnetic field tensor to another. This is the only continuation which is stable to small perturbations in the initial direction and/or the rest mass. By virtue of this transition, the energy density always remains positive. While this section is rather long, the reader may find, at its end, a summary of the results obtained there.

In section 7 we shall apply the results of the former sections to the charged Vaidya solution. In particular, we shall discuss the evolution beyond the hypersurface of vanishing points. If this hypersurface is spacelike, to its future there will be another patch of outgoing charged Vaidya solution. The matching of the two patches is discussed. Finally, in section 8 we discuss the results.

\section{Review of the charged Vaidya solution}

The charged Vaidya geometry is described by the line element

$$
\mathrm{d} s^{2}=2 \mathrm{~d} r \mathrm{~d} v-F(v, r) \mathrm{d} v^{2}+r^{2}\left(\mathrm{~d} \theta^{2}+\sin ^{2} \theta \mathrm{d} \phi^{2}\right)
$$

where

$$
F(v, r)=1-2 m(v) / r+e^{2}(v) / r^{2} .
$$

The massless particles are assumed to move along the ingoing null worldlines $v=$ constant. The two arbitrary functions $m(v)$ and $e(v)$ are, respectively, the mass and electric charge at the advanced time $v$. Accordingly, the only non-vanishing components of the spherically symmetric electromagnetic field $F_{\alpha \beta}$ are $F_{r v}=-F_{v r}=e(v) / r^{2}$. The energy-momentum tensor $T^{\alpha \beta}$ includes two parts:

$$
T^{\alpha \beta}=M^{\alpha \beta}+E^{\alpha \beta} \text {. }
$$

Here, $E^{\alpha \beta}$ is the standard energy-momentum of the electromagnetic field. The matter contribution to the energy-momentum, $\boldsymbol{M}^{\alpha \beta}$, has the following form:

$$
M^{\alpha \beta}=\rho k^{\alpha} k^{\beta}
$$

where $p$ is a scalar and $k^{\alpha}$ is in general a null vector, $k^{\alpha} k_{\alpha}=0$, tangent to the 4-momentum. In particular, for the situation discussed here, $k^{\alpha}$ is tangent to the lines $v=$ constant. Insertion of equation (2) into the Einstein equations yields

$$
\boldsymbol{M}_{\alpha \beta}=\left(4 \pi r^{2}\right)^{-1}[\dot{m}-e \dot{e} / r] \delta_{\alpha}^{v} \delta_{\beta}^{v}
$$

where a dot denotes $\partial / \partial v$.

According to the standard interpretation $[2,3]$, the motion of the massless particles is regarded as geodesic, that is

$$
\bar{k}_{\alpha}=-\delta_{\alpha}^{\nu} \text {. }
$$


Hereafter, the overbar refers to the geodesic motion interpretation. From equation (6) one then obtains for $\rho$ :

$$
\bar{\rho}(r, v)=\left(4 \pi r^{2}\right)^{-1}[\dot{m}-e \dot{e} / r] .
$$

An observer who moves radially with a timelike 4-velocity $u_{\mathrm{ob}}^{\alpha} \equiv \mathrm{d} x_{\mathrm{ob}}^{\alpha} / \mathrm{d} \tau$, where $\tau$ is the observer proper time, will measure an energy density

$$
T_{\mathrm{ob}}=M_{\mathrm{ob}}+(1 / 8 \pi)\left(e^{2} / r^{4}\right)
$$

where $M_{\mathrm{ob}}$, the measured energy density of the null luid, is given by

$$
M_{\mathrm{ob}}=\bar{\rho}\left(\bar{k}_{\alpha} u_{\mathrm{ob}}^{\alpha}\right)^{2}=\bar{\rho}\left(\mathrm{d} v_{\mathrm{ob}} / \mathrm{d} \tau\right)^{2} .
$$

We assume $\dot{m} \geqslant 0$; otherwise $\bar{\rho}$ is negative for large $r$ values. Now, if $e \dot{e}$ is also positive, there is a critical $r$ value,

$$
r_{c}(v) \equiv e \dot{e} / \dot{m}>0
$$

such that for $r<r_{\mathrm{c}}(v) \bar{\rho}$ is negative. In this region, $M_{\mathrm{ob}}$ is negative. An observer with sufficiently large $\mathrm{d} v_{\mathrm{ob}} / \mathrm{d} \tau$ will measure there a total energy density $T_{\mathrm{ob}}<0$.

With the line element of equation (2), the radial null geodesics $v=$ constant extend all the way from $r=\infty$ down to $r=0$. Consequently, with the assumption of geodesic motion (equation (7)), one ends up with the conclusion that the null fluid moves on the lines $v=$ constant up to $r=0$. If correct, this would lead to negative locally measured energy density in the domain $r<r_{\mathrm{c}}(v)$.

\section{The equation of motion}

Before we analyse the special case of spherical flow, it is important to find the appropriate, general EOM for charged massless particles. In principle, this EOM is to be derived from the field equations. Substituting the expression for the energymomentum tensor, equations (4) and (5), into the conservation equation

$$
T_{; \beta}^{\alpha \beta}=0
$$

and using the Maxwell equations, one obtains

$$
F_{\beta}^{\alpha} J_{e}^{\beta}=\left(\rho k^{\alpha} k^{\beta}\right)_{; \beta}=k^{\alpha}\left(\rho k^{\beta}\right)_{; \beta}+\rho k^{\beta} k_{; \beta}^{\alpha}
$$

where $J_{\mathrm{e}}^{\alpha}$ denotes the electric current. The first step is to show that in general the charged massless particles will not move on geodesics. If $k^{\alpha}$ were to satisfy the geodesic equation $k^{\beta} k_{; \beta}^{\alpha}=0$, then equation (13) would imply

$$
F^{\alpha}{ }_{\beta} J_{\mathrm{e}}^{\beta}=k^{\alpha}\left(\rho k^{\beta}\right)_{; \beta} .
$$

Since the electric current originates from the flow of the charged particles, $J_{\mathrm{e}}^{\alpha}$ must be parallel to $k^{\alpha}$. With equation (14), this is possible only if $k^{\alpha}$ is an eigenvector of $F^{\alpha}{ }_{\beta} \dagger$. The matrix $F^{\alpha}{ }_{\beta}$ has just two null eigenvectors (with different eigenvalues). Therefore, a geodesic motion would be consistent with the field equations only if the electromagnetic field and the initial 4-momentum satisfy the following requirements: (i) the integral curves of the null vector field $U^{\alpha}(X)$ coincide with null geodesics, where $U^{\alpha}(X)$ is a null eigenvector of $F_{\beta}^{\alpha}(X)$, and (ii) the initial 4-momentum is everywhere tangent to this vector field.

t More generally, for any null worldline the tangent vector may be scaled as a geodesic tangent only if the acceleration vector $k^{\beta} k_{; \beta}^{\alpha}$ is proportional to $k^{\alpha}$. 
For a generic electromagnetic field $F_{\beta}^{\alpha}(X)$, or generic initial 4-momentum, at least one of these conditions is violated, and the particle's motion cannot be geodesic.

Thus, the fundamental equation of motion must include a Lorentz force. Such an EOM can be obtained easily from the well known EOM of a massive charged particle:

$$
\mu u^{\beta} u_{: \beta}^{\alpha}=q F^{\alpha}{ }_{\beta} u^{\beta}
$$

where $\mu$ and $q$ are, respectively, the rest mass and the electric charge of the particle. It is convenient to write this equation in the form $p^{\beta} p^{\alpha}{ }_{i \beta}=q F^{\alpha}{ }_{\beta} p^{\beta}$, where $p^{\alpha} \equiv \mu u^{\alpha}$ is the 4-momentum of the particle. The rest mass does not appear in this equation, so one can immediately apply it to a massless particle. We expect, therefore, that the EOM of massless charged particles will be of the form

$$
k^{\beta} k_{; \beta}^{\alpha}=q F^{\alpha}{ }_{\beta} k^{\beta}
$$

where $k^{\alpha}$ is the null 4-momentum.

It still remains to derive equation (16) from the field equations. However, $k^{\alpha}$ is not completely determined by the energy-momentum tensor: from equation (5), $\boldsymbol{M}^{\alpha \beta}$ determines the direction of $k^{\alpha}$, not its magnitude; hence, a complete determination of $k^{\alpha}$ would require an additional constraint. In the following, we shall adopt the usual hydrodynamic point of view, and interpret $\rho$ as a (generalized) number-density scalar. We shall think of the null fluid as a stream of discrete massless particles; hence, a conserved null current $J^{\alpha}$ is associated with the flow; that is

$$
J_{; \alpha}^{\alpha}=0 \text {. }
$$

The magnitudes of $\rho$ and $k^{\alpha}$ are now determined by the additional constraint $J^{\alpha} \equiv \rho k^{\alpha} \dagger$. Similarly, if the particles are charged, there is a conserved electric current $J_{\mathrm{e}}^{\alpha}$, parallel to $k^{\alpha}$, and we define the (generalized) charge-density scalar $\rho_{\mathrm{e}}$ by $J_{\mathrm{e}}^{\alpha}=\rho_{\mathrm{e}} k^{\alpha}$. Since both $J^{\alpha}$ and $J_{\mathrm{e}}^{\alpha}$ satisfy the same continuity equation (with the same 4-momentum $k^{\alpha}$ ), the ratio $q \equiv J_{\mathrm{e}}^{\alpha} / J^{\alpha}=\rho_{\mathrm{e}} / \rho$ is conserved along the orbit of the particle. The scalar $q$ is the 'specific charge'-the charge per particle. The conservation of $q$ reflects the fact that each particle carries its own electric charge.

Substituting equation (17) in equation (13), we finally obtain the desired equation of motion, equation (16).

It is often useful to parametrize the null orbits by some parameter $\lambda$. As in the case of neutral massless particles, a natural parametrization is obtained by requiring $k^{\alpha}=$ $\mathrm{d} x^{\alpha} / \mathrm{d} \lambda$. To avoid confusion with the affine parameter, which is used to parametrize null geodesics, we shall call $\lambda$ the Lorentz parameter. Note that when the Lorentz force vanishes, the orbits obtained from equation (16) are just null geodesics, and $\lambda$ becomes the standard affine parameter.

\section{Positivity of energy density and vanishing points}

The observed fluid energy density is given by

$$
M_{o b}=\rho\left(k_{\alpha} u_{o b}^{\alpha}\right)^{2}
$$

t One can always formally construct such a conserved current vector $J^{a}$, parallel to the direction of $k^{\alpha}$ (as determined from $\boldsymbol{M}^{\alpha \beta}$ ), in the following way: define $K^{\alpha}$ to be the future-directed vector such that $K^{\alpha} K^{\beta}=M^{\alpha \beta}$. Then, find the orbits $x^{\alpha}(\lambda)$ by solving the equation $\mathrm{d} x^{\alpha} / \mathrm{d} \lambda=K^{\alpha}$. Along each orbit define $s$ by $\mathrm{d} \ln (s) / \mathrm{d} \lambda=-K^{\alpha}{ }_{\alpha}$. The desired current vector is then $J^{\alpha}=s K^{\alpha}$. By this construction, $J^{\alpha}$ is determined up to a renormalization factor which is constant along the orbit but may vary from orbit to orbit. Such a renormalization of $J^{\alpha}$ would induce a corresponding renormalization of $k^{\alpha}$ and $\rho$ along that orbit. 
(see equation (5)). The interpretation of this quantity is simple. The measured number density (namely, number of particles per unit of three-dimensional volume) $n_{\mathrm{ob}}$ is given by

$$
n_{\mathrm{ob}}=J_{\alpha} u_{\mathrm{ob}}^{\alpha}=\rho\left(k_{\alpha} u_{\mathrm{ob}}^{\alpha}\right)
$$

and the measured energy of each particle is equal to its observed wavenumber, $k_{\alpha} u_{\mathrm{ob}}^{\alpha}$.

In view of this interpretation, one would naturally expect that the energy density of charged null fluid be generally positive. This can be seen as follows. (i) The measured number density $n_{o b}$ must be positive—provided that it was positive initially (one can show this explicitly by considering an infinitesimally small three-dimensional volume element on the initial slice and letting its boundary 'flow' with the particles. The total number of particles in this volume is initially positive and is conserved). (ii) The 4-momentum vector $k^{\alpha}$ is future-directed (as we shall see) and $u_{\mathrm{ob}}^{\alpha}$ is future-directed. This implies that $k_{\alpha} u_{\mathrm{ob}}^{\alpha}$, the energy of each particle, is positive.

While it is physically plausible that $k^{\alpha}$ is future-directed, the question remains whether, mathematically, future directivity is respected by the EOM. From the light cone structure it is clear that, in order to switch from future-directed to past-directed, $k^{\alpha}$ must vanisht. We shall call such a point, where $k^{\alpha}=0$, a vanishing point. Simple arguments indicate that vanishing points do exist in generic charged null fluid models, though only on a zero-measure set of orbits: since $k^{\alpha}$ is null, the requirement $k^{\alpha}=0$ is a set of three constraints. From a simple counting of degrees of freedom, we can expect that for a generic initial 4-momentum the vanishing points (if they exist) will span isolated one-dimensional curvest. In highly symmetric models, however, the vanishing points can form a hypersurface. For instance, the hypersurface $r=r_{c}(v)$, which often exists in the charged Vaidya solution, is such a hypersurface of vanishing points.

Whether it is a one-dimensional curve in a generic flow or a higher-dimensional set of points in more symmetric flows, we realize that in general vanishing points do exist; hence, in order to answer the question of positivity of $k^{\alpha}$, we have to understand the behaviour of the fluid orbits at the vanishing points. For that purpose, it is useful to regard the geometry and electromagnetic field as given a priori, and to study the motion of massless charged test particles in such a background. This is allowed, as we already used the field equations to derive the EOM. In the following two sections we shall use this framework of test particles in a given background: section 5 for a spherical model and section 6 for a generic flow. Indeed, after one has obtained the rules of how to continue the orbits beyond the vanishing point, the next step is to plug them back into the field equations, in order to determine the evolution (of the geometry and the electric field) beyond the hypersurface of vanishing points. This will be done in section 7 for the spherically symmetric case, the charged Vaidya solution.

\footnotetext{
† By a past-directed $k^{\alpha}$ we do not mean an orbit that turns to the past: we insist that the orbit goes monotonically from the past to the future; still the possibility exists that the 4-momentum, as defined by the EOM, becomes past-directed. In such a case, the parameter $\lambda$ must switch from increasing with time to decreasing with time. If there is such a transition from increasing to decreasing $\lambda$, it must occur at the vanishing point, where, as will be shown later, $\lambda$ is infinite.

$¥$ A careful counting of degrees of freedom must take into account the qualitative local behaviour of the orbits near a vanishing point, which is discussed in section 6 . From the discussion there one obtains the same results (see e.g. equation (29), which implies that an orbit which admits a vanishing point must satisfy two extra constraints: $k^{x}=k^{y}=0$ in the terminology used there).
} 


\section{The spherically-symmetric case}

In this section we shall analyse the radial motion of a massless, charged test particle in the Reissner-Nordstrom spacetime. In particular, we shall focus attention on the behaviour near the vanishing point. In Schwarzschild coordinates, the line element takes the form

$$
\mathrm{d} s^{2}=-F \mathrm{~d} t^{2}+F^{-1} \mathrm{~d} r^{2}+r^{2}\left(\mathrm{~d} \theta^{2}+\sin ^{2} \theta \mathrm{d} \phi^{2}\right)
$$

where $F(r)$ is given by equation (3), but with constant $m$ and $e$ parameters. The electric field is given by $F_{\mathrm{rt}}=-F_{\mathrm{tr}}=e / r^{2}$. It is straightforward to solve the EOM, equation (16), for $k_{t}$ :

$$
k_{t}=-\left(k_{\infty}-e q / r\right)
$$

The free parameter $k_{x}$ represents the energy of the particle, as measured by a distant, static observert. Note that a massive test particle obeys a similar equation: $\mu u_{t}=$ $-\left[\left(-\mu u_{t}\right)_{\infty}-e q / r\right]$. Here, again, $\left(-\mu u_{t}\right)_{\infty}$ is the energy of the particle as measured at infinity.

For a radial orbit, the normalization condition yields

$$
k^{r}= \pm k_{t} \text {. }
$$

Initially, the infalling particle satisfies

$$
k^{r}=k_{\mathrm{t}}<0 \text {. }
$$

For $e q>0$, which we consider here, there is a vanishing point at

$$
r=r_{\mathrm{c}} \equiv e q / k_{\infty} \text {. }
$$

At this point both $k^{r}$ and $k_{\mathrm{r}}$ vanish. One can show that $r=r_{\mathrm{c}}$ is reached in finite $t$, $t=t_{c}$, though the Lorentz parameter diverges there (from equations (19) and (20), $\lambda$ is approximately proportional to $\ln \left(r-r_{c}\right)$ ). We denote the vanishing point by $X_{c} \equiv$ $\left(t_{\mathrm{c}}, r_{\mathrm{c}}, \theta, \phi\right)$. What happens after that? From equation (16) it is clear that the vanishing point is a critical point and the EOM just reads $0=0$ there. To determine the continuation, we must look for solutions of equation (16) which, in their past, terminate at $r=r_{\mathrm{c}}$ with $k^{r}=k^{t}=0$. There are two such solutions, corresponding to the two possible signs on the right-hand side of equation $(20)\left(k_{f}\right.$ is still given by equation (19), with the original $k_{\infty}$ ). That is, mathematically there are two possible continuations beyond the vanishing point:

$$
\begin{array}{ll}
\text { the non-bouncing continuation: } & k^{r}=k_{l} \\
\text { the bouncing continuation: } & k^{r}=-k_{\mathrm{r}} .
\end{array}
$$

For the non-bouncing continuation, $\mathrm{d} r / \mathrm{d} t$ preserves its sign at $X_{\mathrm{c}}$ (it is assumed here that the vanishing point is not located exactly on the horizon). This means that the orbit continues along the same ingoing null direction, and $r$ continues to decrease with time. Equations (19) and (20a) then imply that both $k^{r}$ and $k^{t}$ change sign. We find, therefore, that for the non-bouncing continuation $k^{\alpha}$ becomes past-directed, and the

+ We define the 'direction' of the coordinate $t$ such that $\mathrm{d} t$ is future-directed whenever $t$ is timelike (i,e. outside the black hole and inside the inner horizon). In the intermediate range (between the horizons), where $t$ is spacelike, we define its direction such that $k_{\text {f }}$ preserves its sign at the horizons. With this convention, equation (19) is valid in all regions of the spacetime (with the same $k_{\text {rec }}$ ). 
energy carried by the particles becomes negative (see section 4). Note that this result is independent of the sign of $F$.

For the bouncing continuation, the situation is different: at $X_{\mathrm{c}}, \mathrm{d} r / \mathrm{d} t$ changes sign. This means that the orbit switches now to an outgoing null direction. To analyse the sign of $k^{\alpha}$, it is worthwhile to distinguish between the two different cases.

(i) $F>0$ : the coordinate $t$ is timelike. The change in the sign of $\mathrm{d} r / \mathrm{d} t$ means that $r$ begins to increase. Therefore, $k_{l}$ remains negative (equation (19)) and $k^{\alpha}$ remains future directed.

(ii) $F<0$ : now, the coordinate $r$ is timelike and $r$ continues to decrease after $X_{\mathrm{c}}$. From equations (19) and (20b) one finds that $k_{\mathrm{r}}$ becomes positive but $k^{r}$ preserves its sign. Again, $k^{\alpha}$ remains future directed.

Thus, for the bouncing continuation, and for both cases $F>0$ and $F<0, k^{\alpha}$ remains future-directed and the energy of the particle remains positive.

Which of these continuations is more reasonable from the physical point of view? To answer this question, we recall that the assumption of a pure radial motion is oversimplified. For more realistic initial data, the particles will always have some non-radial 4-momentum component. The magnitude of this tangential component can be described by the conserved angular-momentum parameter $L \equiv k_{\phi}=r^{2} \mathrm{~d} \phi / \mathrm{d} \lambda$ (without loss of generality, we shall consider here equatorial motion, $\theta=\pi / 2$ ). Conservation of $L$ implies that no matter how smali is the initial perturbation, it will always prevent the vanishing of $k^{\alpha}$. Therefore, there is no longer mathematical ambiguity in the determination of the orbit. Moreover, since the 4-momentum cannot vanish, it remains future-directed forever. This simple argument proves that in the limit $L \rightarrow 0$ one does not obtain the radial non-bouncing continuation. To show that one does obtain the radial bouncing continuation in this limit, we shall now briefly analyse the motion of a particle with $L \neq 0$. The angular motion is described by the conserved parameter $L$ and $k_{t}$ is still given by equation (19). The zero-mass condition reads

$$
\left(k^{r}\right)^{2}=k_{t}^{2}-\frac{F}{r^{2}} L^{2}
$$

Here, again, we shall distinguish between the two cases.

(i) $F>0$ : there is a turning point at $r=r_{s}+F^{1 / 2} L / k_{\infty}>r_{\varepsilon}$. The particle then bounces, in a time-symmetric manner, now with positive $k^{r}+$. Since $r=r_{c}$ is not reached, $k^{t}$ preserves its (positive) sign and never vanishes.

(ii) $F<0$ : here, $r=r_{\mathrm{c}}$ is reached, still with negative $k^{r}$. At $r=r_{\mathrm{c}}, k^{t}$ vanishes and changes its sign. However, $k^{r}$ never vanishes.

In both cases, the resultant signs of $k^{r}$ and $k^{t}$ are just the same as in the radial bouncing continuation described above. In the limit $L \rightarrow 0$, the orbit $r(t)$ precisely coincides with the radial bouncing continuation.

On the basis of this perturbation analysis, it appears that the bouncing continuation is the physical one. Consequently, the energy density of the massless particles is always positive.

The analysis here was restricted to the spherically symmetric model. We shall immediately show, however, that the results obtained here are very general and apply for a generic flow of massless charged particles.

t The change in the sign of $k^{r}$ here (as well as that of $k^{\prime}$ in case 2) is not apparent from the above expression for $k^{r}$. One can show it, however, by using the original, second-order, EOM. The situation here is similar to the turning points of Newtonian Keplerian orbits. 


\section{Properties of generic vanishing points}

In this section we shall study the local behaviour of the orbits near a generic vanishing point. One can always choose coordinates which are locally Lorentz at the vanishing point. It is sufficient to study the orbits in an arbitrarily small neighbourhood of the vanishing point; hence, the spacetime curvature and local variations in the electromagnetic field are unimportant for this analysis. We can therefore explore the main features of generic vanishing points by studying the orbits of massless charged particles in fiat spacetime with a constant electromagnetic field, $F_{\beta ; \eta}^{\alpha}=0$. Hereafter, we shall use Lorentz flat-spacetime coordinates $(x, y, z, t)$. Equation (16) then takes the form

$$
\mathrm{d} k^{\alpha} / \mathrm{d} \lambda=q F^{\alpha}{ }_{\beta} k^{\beta}
$$

where the elements of $F^{\alpha}{ }_{\beta}$ are constant (with respect to both space and time).

For a generic, constant electromagnetic field, we can assume that both the electric and magnetic fields are aligned in the $z$ direction. One can generally arrange this with the aid of a Lorentz transformation [5] $\dagger$. Let $E$ and $B$ be the $z$ components of the electric and magnetic fields, respectively, in this Lorentz frame. Without loss of generality, we can assume $q E>0 ; B$ may or may not vanish. Equation (22) separates now into two independent sets of equations:

$$
\frac{\mathrm{d} k^{2}}{\mathrm{~d} \lambda}=-q E k^{1} \quad \frac{\mathrm{d} k^{\prime}}{\mathrm{d} \lambda}=-q E k^{2}
$$

for the $z, t$ components, and

$$
\frac{\mathrm{d} k^{x}}{\mathrm{~d} \lambda}=q B k^{y} \quad \frac{\mathrm{d} k^{y}}{\mathrm{~d} \lambda}=-q B k^{x}
$$

for the $x, y$ components.

Later we will be interested in the effect of a small, non-zero rest mass on the orbits. For that purpose, it is worthwhile to consider the cases of both massive and massless particles in the same formal framework. Thus, we denote by $k^{\alpha}$ the 4-momentum of the particle (for a massive particle, $k^{\alpha}=\mu u^{\alpha}$ ). Then, equation (16) applies to both cases. For a massive particle, we define $\lambda$ as the proper time divided by $\mu$, so that again $k^{\alpha}=\mathrm{d} x^{\alpha} / \mathrm{d} \lambda$. Then, equations (22)-(24) hold for a massive particle as well. In this representation, the dynamics is the same for both cases, and the notation of zero or non-zero mass is merely a statement about the initial value of $\mu^{2}=-k_{\alpha} k^{\alpha}$ (indeed, the EOM conserves this quantity).

Let us denote by $k_{\|}^{\alpha}$ and $k_{\perp}^{\alpha}$ the projections of $k^{\alpha}$ on the $t z$ and the $x y$ planes, correspondingly. From equations (23) and (24), $k_{\|}^{\alpha}$ and $k_{\perp}^{\alpha}$ evolve independently. In particular, both $k_{\perp} \equiv\left(k_{\perp \alpha} k_{\perp}^{\alpha}\right)^{1 / 2}=\left[\left(k^{x}\right)^{2}+\left(k^{l}\right)^{2}\right]^{1 / 2}$ and $k_{\|\|} \equiv\left(-k_{\| \alpha} k_{\|}^{\alpha}\right)^{1 / 2}=$ $\left[\left(k^{t}\right)^{2}-\left(k^{z}\right)^{i}\right]^{1 / 2}$ are conserved independently. The motion in the $x y$ plane is not so important to our discussion, and we shall just briefly mention the following results. In the general case, $B \neq 0$, the particle will move on a circular orbit in the $x y$ plane. Both the radius $R$ of the circle and the 'Lorentz frequency' $\omega_{\lambda}$ (i.e. number of circles per unit Lorentz parameter) are constants of the motion:

$$
R=\frac{k_{\downarrow}}{q B} \quad \omega_{\lambda}=q B .
$$

t The only exception is the case in which the electric and magnetic fields are perpendicular to each other and of the same magnitude. This special case requires a separate treatment. 
In the special case $B=0$, the motion in this plane is linear, and both $k^{x}$ and $k^{y}$ are conserved. The important fact is that in both cases $k_{\perp}$ is conserved.

We will be mainly interested here in the motion in the $z t$ plane. This motion is the most important for understanding the vanishing points. One can easily show that $k^{\prime}=0$ (or $k_{\|}^{\alpha}=0$ ) is a necessary and sufficient condition for a vanishing point. The motion in the $z t$ plane is linked to the transverse motion only through the normalization condition $\mu^{2}=-k_{\alpha} k^{\alpha}$, which reads

$$
\left(k_{\|}\right)^{2}=\left(k_{\alpha}\right)^{2}+\mu^{2}
$$

The general solution of equation (23) is

$$
k_{\|}^{\alpha}=A_{+} V_{+}^{\alpha} \mathrm{e}^{q E \lambda}+A_{-} V_{-}^{\alpha} \mathrm{e}^{-q E \lambda}
$$

where $\boldsymbol{A}_{+}$and $A_{-}$are constants. $V_{+}$and $V_{-}$are constant vectors in the $z t$ space, defined by $\left(V_{ \pm}^{\bar{z}}, V_{ \pm}^{\mathrm{s}}\right)=( \pm 1,1)$. The normalization condition, equation (25), now implies

$$
\boldsymbol{A}_{+} \boldsymbol{A}_{-}=\left(k_{\perp}\right)^{2}+\mu^{2}
$$

According to equation (26) both $A_{+} \mathrm{e}^{q E \lambda}$ and $A_{-} \mathrm{e}^{-q E \lambda}$ must tend to zero at the vanishing point. There are two possibilitiest:

$$
A_{+}=0 \quad \lambda \rightarrow \infty
$$

or

$$
A_{-}=0 \quad \lambda \rightarrow-\infty .
$$

From equation (27), a necessary and sufficient condition for the existence of a vanishing point in the orbit (in either the past or the future) is

$$
k_{\perp}=\mu=0 \text {. }
$$

The orbit $x^{\alpha}(\lambda)$ is obtained by integrating equation (26) once again:

$$
\begin{aligned}
& t(\lambda)=\frac{1}{q E}\left(A_{+} \mathrm{e}^{q E \lambda}-A_{-} \mathrm{e}^{-q E \lambda}\right)+t_{\mathrm{c}} \\
& z(\lambda)=\frac{1}{q E}\left(A_{+} \mathrm{e}^{q E \lambda}+A_{-} \mathrm{e}^{-q E \lambda}\right)+z_{\mathrm{c}}
\end{aligned}
$$

where $t_{\mathrm{c}}$ and $z_{\mathrm{c}}$ are constants of integration. An orbit with a vanishing point satisfies $x=$ constant $\equiv x_{c}, y=$ constant $\equiv y_{c}$. Thus, the vanishing point is located at finite flatspacetime coordinate values $X_{\mathrm{c}}^{\alpha}=\left(x_{\mathrm{c}}, y_{\mathrm{c}}, z_{\mathrm{c}}, t_{\mathrm{c}}\right)$, not at infinity. However, the Lorentz parameter is infinite there.

Initially, the 4-momentum is future-directed, and $\lambda$ must increase with time as the particle approaches the vanishing point. Therefore, the approach to the vanishing point (from the past) is described by equation (28a), with positive $A_{-}$. However, due to the divergence of $\lambda$, all the $\lambda$ derivatives of $k^{\alpha}$ vanish there (as well as $k^{\alpha}$ itself). Therefore, the constants $A_{+}$and $A_{-}$may be changed at the vanishing point without violating the EOM (equation (22) or equation (16)) - - provided that either equation (28a) or equation $(28 b)$ is satisfied. This introduces an ambiguity in the continuation of the orbit there.

† The trivial possibility $A_{+}=A_{-}=0$ is physically meaningless. 
Considering only orbits that go from past to future, there are two possible mathematical continuations beyond $X_{c}^{\alpha}$.

(i) Equation (28a), with negative $A_{-}$(the non-bouncing continuation).

(ii) Equation (28b) with positive $A_{+}$(the bouncing continuation).

To express this result in a more direct, invariant manner, we define the two null 4-vectors $U_{+}^{\alpha}$ and $U_{-}^{\alpha}$ by $U_{*}^{\alpha} \equiv(0,0, \pm 1,1)$. Note that $U_{+}^{\alpha}$ and $U_{-}^{\alpha}$ are the two real, null eigenvectors of $F^{\alpha}{ }_{\beta}$. We find, that the orbit approaches the vanishing point along the null eigenvector $U_{-}^{\alpha}$. Then, mathematically, the orbit may either continue along the same eigenvector (the non-bouncing continuation), or switch to the other null eigenvector, $U_{+}^{\alpha}$ (the bouncing continuation).

The negative sign of $A_{-}$in the non-bouncing continuation means that $k^{\alpha}$ is past-directed, which implies negative energy density. Thus, we have just enough mathematical freedom to impose the requirement of positive energy density. This reasonable requirement would exclude the non-bouncing continuation and determine the continuation uniquelyt. We shall immediately show that a stability analysis also identifies the bouncing continuation as the more physical one.

To study the stability of the orbit against small perturbations in the initial $k^{\alpha}$, we consider two types of perturbations: a small rest-mass $\mu \neq 0$, and a small transverse component, $k_{\perp} \neq 0$. Such perturbations will remove the vanishing point, making it possible to calculate the orbit without any mathematical ambiguity. Then, we will focus attention on the limit $\mu, k_{\perp} \rightarrow 0$, keeping $q$ and $E$ constant. As a reference, we consider a particle with $\mu=k_{\perp}=0$, which at $t=0$ (and, say, $\lambda=0$ ) is located at $x=y=z=0$, with $A_{-}=A>0$. Equation (27) then implies $A_{+}=0$, and hence $k_{\|}^{\alpha}=A V_{\infty}$. Such an orbit has a vanishing point at

$$
x=y=0 \quad z=z_{\mathrm{c}}=-t_{\mathrm{c}} \quad t=t_{\mathrm{c}}
$$

where $t_{\mathrm{c}} \equiv A / q E$. Then, according to the bouncing continuation, the particle switches to the $V_{+}$direction. At $t=2 t_{\mathrm{c}}$ the particle will be located again at $x=y=z=0$. According to the non-bouncing continuation, at $t=2 t_{\mathrm{c}}$ the reference particle will be located at $x=y=0, z=2 z_{c} \neq 0$, with $k^{\alpha}$ in the (negative) $V_{-}$direction.

Let us now introduce the perturbation. We still assume $A_{-}=A>0$, but now we have from the normalization condition, equation (27),

$$
A_{+}=\left(k_{\perp}^{2}+\mu^{2}\right) A^{-1} \equiv \varepsilon>0
$$

and we think of $\varepsilon$ as a small number. The initial motion is given, therefore, by $k_{\|}^{\alpha}=A V_{-}^{\alpha}+\varepsilon V_{+}^{\alpha}$. From equation (26) one can see that at $\lambda=\lambda_{\mathrm{c}} \equiv \ln (A / \varepsilon) / 2 q E, k^{2}$ vanishes. At this moment $k^{\prime}$ approaches a minimal value, $k^{\prime}=2 \sqrt{\varepsilon A}$. It is trivial to show that in the limit $\mu, k_{\perp} \rightarrow 0$ the turning point $\lambda=\lambda_{\mathrm{c}}$ becomes the vanishing point of the reference particle, that is

$\lim _{\varepsilon \rightarrow 0} X^{\alpha}\left(\lambda=\lambda_{c}\right)=\left(0,0,-t_{c}, t_{c}\right) \quad \lim _{\varepsilon \rightarrow 0} k^{\alpha}\left(\lambda=\lambda_{c}\right)=0 \quad \lim _{\varepsilon \rightarrow 0} \lambda_{c}=\infty$.

It remains to compare the motion beyond the turning point to that of the reference particle, beyond its vanishing point. Using equation (26) one observes that at $\lambda>\lambda_{\mathrm{c}}$ the motion is inverted, in a time-symmetric manner: $k^{z}$ becomes negative, while $k^{t}$

t The remaining degree of freedom related to the magnitude of $A_{+}$is not significant, because one can always get rid of it by a shift in $\lambda$. 
increases again. Then, at $\lambda=2 \lambda_{c}, z$ vanishes again and $k_{\sharp}^{\alpha}=A V_{+}^{\alpha}+\varepsilon V_{-}^{\alpha}$. In the limit $\mu, k_{\perp} \rightarrow 0$ one obtains

$$
\lim _{\varepsilon \rightarrow 0} X^{\alpha}\left(\lambda=2 \lambda_{c}\right)=\left(0,0,0,2 t_{c}\right) \quad \lim _{\varepsilon \rightarrow 0} k^{\alpha}\left(\lambda=2 \lambda_{c}\right)=A V_{+}^{\alpha} .
$$

We see that both the location and 4-momentum of the perturbed orbit precisely agree, in the limit of vanishing perturbations, with the bouncing continuation of the unperturbed reference orbit. The perturbation analysis shows, therefore, that the bouncing continuation is the correct one, since it matches smoothly to the case $\mu, k_{\alpha} \neq 0$.

To conclude, from studying the motion of charged test particles in flat spacetime with a constant electromagnetic field we have learned the following properties of generic vanishing points.

(i) A given vanishing point can be approached only from a direction that is parallel to one of the two real, null eigenvectors of $F^{\alpha}{ }_{\beta}$.

(ii) The vanishing point is located at finite values of local Lorentz coordinates, not at infinity. However, $\lambda$ diverges and there is an ambiguity in the solution of the EOM there. Mathematically, there are two possible continuations, corresponding to the two real, null eigendirections of $F^{\alpha}{ }_{\beta}$. In the non-bouncing continuation, the orbit continues along the same eigendirection. In the bouncing continuation, the orbit switches to the other null eigendirection.

(iii) In the bouncing continuation the energy density is always positive; in the non-bouncing continuation it is always negative.

(iv) A perturbation, in the form of a small deviation of the initial 4-momentum from the null eigendirection and/or a small non-zero rest mass, removes the vanishing point and prevents the mathematical ambiguity. In the limit of zero perturbations, one recovers again the bouncing continuation. Consequently, the bouncing continuation is the natural one and the energy density of charged null fluid is always positive.

\section{Implications for the charged Vaidya solution}

In this section we shall apply the formalism of the previous sections to the charged Vaidya solution. The fluid energy-momentum is given by

$$
M^{\alpha \beta}=\left(4 \pi r^{2}\right)^{-1}(\dot{m}-e \dot{e} / r) \delta_{r}^{\alpha} \delta_{\mathrm{r}}^{\beta}
$$

(see equation (6)). The number-density current $J^{\alpha}$ is tangent to $k^{\alpha}$; hence, it is proportional to $\delta_{r}^{\alpha}$. The continuity equation, equation (17), then yields

$$
J^{\alpha}=\frac{-\phi(v)}{4 \pi r^{2}} \delta_{r}^{\alpha}
$$

The arbitrary function $\phi(v)$ represents the flux of particles at the retarded time $v$. Comparing equations (35) and (36), one obtains

$$
k^{\alpha}=-\frac{1}{\phi}(\dot{m}-e \dot{e} / r) \delta_{r}^{\alpha}
$$

and

$$
\rho=\frac{\phi^{2}}{4 \pi r^{2}(\dot{m}-e \dot{e} / r)}
$$


The energy of the particles as measured by a distant, static observer is obtained from equation (37):

$$
k_{\infty}(v)=-\lim _{r \rightarrow \infty} k^{r}(r, v)=\dot{m} / \phi
$$

From the Maxwell equations, one obtains

$$
J_{e}^{\alpha}=\frac{-\dot{e}}{4 \pi r^{2}} \delta_{r}^{\alpha}
$$

Comparing equations (36) and (40), one finds that the charge per particle is

$$
q(v)=\dot{e} / \phi
$$

With equations (37), (39) and (41), one can write $k^{r}$ as

$$
k^{r}=-\left(k_{\infty}-e q / r\right)
$$

(compare with equations (19) and (20)).

From equations (39), (41) and (42), the hypersurface of vanishing points is located at

$$
r=r_{\mathrm{c}}(v)=e q / k_{\infty}=e \dot{e} / \dot{m}
$$

(compare with equations (ii) and (2ij)).

In sections 5 and 6 we studied the behaviour of orbits at the vanishing points in a simplified framework. We considered the motion of test particles in a given geometry and electromagnetic field. The next logical step is to plug the results back into the field equations, in order to predict the evolution of the electromagnetic field and the geometry beyond the hypersurface of vanishing points. We shall consider here the case of a spacelike hypersurface $r=r_{c}(v)$, and denote this hypersurface by $\Sigma$. At this hypersurface, the particles switch from ingoing to outgoing null orbits. Consequently, to the future of $\Sigma$ there will be a piece of outgoing charged Vaidya solution, with null fluid moving along outgoing null orbits $u=$ constant (see figure 1 ). The line element

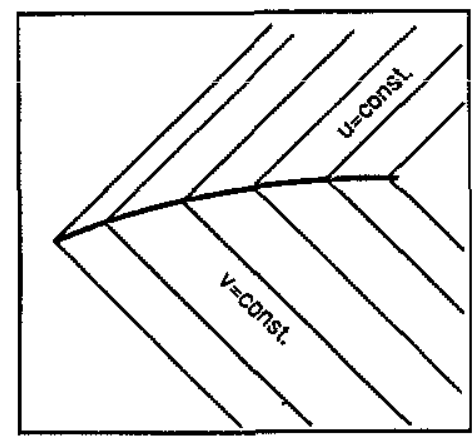

Figure 1. A conformal diagram of a section $\theta, \phi=$ constant in a spherically symmetric spacetime with a radially-moving charged null fluid for a spacelike hypersurface $\Sigma$. This diagram shows the orbits of the fluids shells in the vicinity of the hypersurface $\Sigma$. The thin full lines are the fluid null orbits and the bold curve is $\Sigma$.

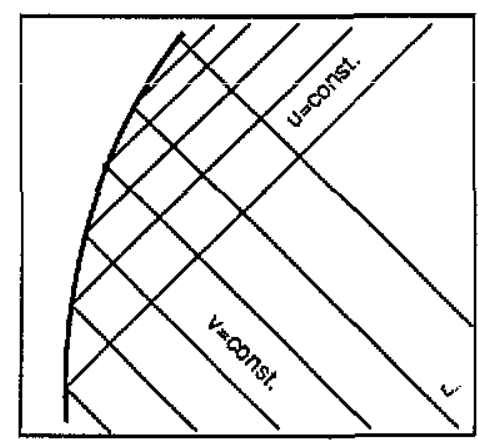

Figure 2. As figure 1 for a timelike hypersurface $\Sigma$. 
has the same form as that of the ingoing charged Vaidya solution, except that now $v$ is replaced by $u \dagger$ :

$$
\mathrm{d} s^{2}=2 \mathrm{~d} r \mathrm{~d} u-F(u, r) \mathrm{d} u^{2}+r^{2}\left(\mathrm{~d} \theta^{2}+\sin ^{2} \theta \mathrm{d} \phi^{2}\right)
$$

where

$$
F(u, r)=1-2 m(u) / r+e^{2}(u) / r^{2} .
$$

Comparing the angular part of the metric on both sides of $\Sigma$, we find that $r$ must be continuous there. Moreover, as $\Sigma$ is spacelike, it cannot be endowed with a massive and/or charged thin layer. We therefore must insist on a $C^{1}$ matching for both the metric and the electric 4-potential. Consequently, $m, e$ and $F$ are also continuous at $\Sigma$. That is,

$$
m(u)=m(u(v)) \quad e(u)=e(u(v))
$$

and the matching is uniquely determined by the function $u(v)$ that relates $u$ to $v$ at $\Sigma$. This function is obtained by comparing the proper distance along $r=r_{\mathrm{c}}(v)$, as expressed in both $(r, v)$ and $(r, u)$ coordinates. One finds that

$$
\frac{\mathrm{d} u(v)}{\mathrm{d} v}=\frac{1}{F(v)}\left[2 \frac{\mathrm{d} r_{\mathrm{c}}}{\mathrm{d} v}-F(v)\right]
$$

where $F(v) \equiv F\left(v, r_{c}(v)\right)$. By a straightforward computation of the extrinsic curvature on both sides of $\Sigma$, one can show that the matching described here is indeed $C^{1}$ with respect to both the metric and the electric 4-potential. This is the only possible $C^{1}$ matching of an outgoing charged Vaidya solution to the ingoing one at $\Sigma$. Moreover, it appears that the matching described here is even $C^{2}$ with respect to the metric. This is because both the Weyl and the Ricci tensors are continuous there. (However, the electric current is in general discontinuous at $\Sigma$; hence, the 4-potential is not $C^{2}$ there.)

The transition to outgoing null orbits prevents the energy density from becoming negative, because, in the outgoing phase, the energy density is no longer described by equations (8) and (10). It still remains to calculate the energy density in this phase and to show that it is always positive. Due to the similarity in the line elements of the two phases, in the outgoing phase the observed energy density $M_{o b}$ is obtained from equation (10) by a replacementt of $v$ and $u$, that is,

$$
M_{\mathrm{ob}}=\bar{\rho}_{\mathrm{u}}\left(\mathrm{d} u_{\mathrm{ob}} / \mathrm{d} \tau\right)^{2}
$$

where

$$
\bar{\rho}_{u} \equiv\left(4 \pi r^{2}\right)^{-1}\left(\frac{\mathrm{d} m}{\mathrm{~d} u}-\frac{e}{r} \frac{\mathrm{d} e}{\mathrm{~d} u}\right)=\left(4 \pi r^{2}\right)^{-1}\left[\dot{m}-\frac{e \dot{e}}{r}\right] \frac{\mathrm{d} v}{\mathrm{~d} u}
$$

(compare with equation (8)). Note that the term in the brackets on the right-hand side of equation (47) is always positive. This results from the requirement that $\Sigma$ be spacelike. Therefore, the sign of $\mathrm{d} u / \mathrm{d} v$ is the same as that of $F$ at the vanishing point. Also, the sign of the brackets on the right-hand side of equation (49) is the same as that of $r-r_{\mathrm{c}}$. Now, from the discussion in section 5 it is clear that if $F$ is positive at the vanishing point, $r-r_{c}$ remains positive beyond it. By contrast, if $F$ is negative there, $r-r_{c}$ becomes negative. We find, therefore, that the right-hand side of equation (49) is always positive. This ensures the positivity of $M_{\mathrm{ob}}$.

$\dagger$ In this line element, the coordinate $u$ is the 'minus advanced time'. 
The situation is more complicated if the hypersurface $\Sigma$ is timelike (see figure 2). Here, there is a shell crossing, a mixing of the outgoing and ingoing flows. The future domain of dependence of $\Sigma$ cannot be described by the charged Vaidya solution. A more general solution, which includes two simultaneous null flows, is required in this case, but as yet the analytic form of such a solution is unknown.

\section{Discussion}

We have shown that, in general, for massless charged particles the geodesic equation is incompatible with the Maxwell-Einstein equations. The correct equation of motion includes a Lorentz force term. In the charged Vaidya solution, due to this force, the 4-momentum $k^{\alpha}$ vanishes at $r_{c}$ (the radius where the fluid energy density vanishes). Then, the Lorentz force pushes the particles and accelerates them along outgoing null geodesics. As a result, the energy density is always positive. This result is not restricted to spherical symmetry. From the discussion in sections 4 and 6 it is clear that charged null fluid always satisfies the weak energy condition-provided that it satisfied it on the initial slice.

There remains one delicate issue which requires some more discussion: the mathematical ambiguity in the continuation of the orbits at the vanishing points. To make things conceptually clear, let us discuss this ambiguity in the framework of spherical symmetry. At the vanishing point $r=r_{\mathrm{c}}$, there are two possible ways to continue the orbit in accordance with the EOM. One is the bouncing continuation described above; the other is the non-bouncing continuation, in which the particle continues along its original ingoing null direction. The non-bouncing continuation results in a violation of the weak energy condition.

However, there is an obvious physical reason to adopt the bouncing continuation. As shown in section 5 , in the Reissner-Nordstrom geometry, the mathematical ambiguity only exists when the orbits (of the massless test particles) are strictly radial. Any small tangential 4-momentum component will destroy the vanishing point and the associated ambiguity. With such a non-radial 4-momentum component one never obtains negative energy density. Moreover, in the limit of vanishing non-radial components (which one can compute without any ambiguity) one always recovers the strictly radial bouncing continuation.

We have to keep in mind that in a physical system the flow is never strictly radial. The massless particles will always have some, even if very small, tangential 4-momentum component. Indeed, in principle we can prepare the initial data such that the flow will be radial to any desired level of approximation, and this is the logical justification for using the model of purely radial flow. Therefore, we are mainly interested in this strictly radial flow model as a limiting case. And the limiting process uniquely selects the bouncing continuation.

Another way to remove the mathematical ambiguity is by considering, instead of tangential 4-momentum components, a small, non-zero rest mass. One obtains the same result: the energy density can never become negative. In the limit of zero rest mass, one recovers again the bouncing continuation. Therefore, whenever the null fluid is considered as a simplified model for an extremely relativistic fluid of massive particles, one does not even need the stability argument to discard the non-bouncing continuation.

As shown in section 6, the same situation exists in the generic (non-spherical) model. For a given geometry and electromagnetic field, and a given initial location of 
the massless particle, only a discrete set of initial 4-momentum directions (typically only one direction) will result in a vanishing point. Any perturbation, in either the initial 4-momentum direction or the rest mass, will destroy the vanishing point and avoid the mathematical ambiguity. By taking the limit of zero perturbations, one uniquely recovers the bouncing continuation.

In view of these arguments, it seems that we must adopt the bouncing continuation at the vanishing points. Consequently, we conclude that charge null field never violates the weak energy condition.

After this work was completed, W Israel informed me of two recent works on the evolution of null thin layers in general relativity $[6,7]$. These works arrive at similar conclusions to this paper, but in the framework of thin massive shells rather than a continuous fluid. If a null shell moves against a force (e.g. the Lorentz force), its locally measured energy density may vanish at some stage. At this point, the null generators of the shell may switch to a new null direction. This transition prevents the violation of the weak energy condition. The present work extends that result from null thin layer to a continuous fiuid. In addition, the stability analysis presented here indicates that (at least in the continuum case) the bouncing continuation is the physically correct one.

\section{Acknowledgments}

I would like to thank $\mathrm{K}$ Thorne and $\mathrm{W}$ Israel for interesting discussions and helpful comments. This research was supported by a Richard Chace Tolman fellowship, a Chaim Weizmann fellowship, a Fulbright grant, and NSF Grant No AST-8817792.

\section{References}

[1] Bonnor W D and Vaidya P C 1970 Gen. Rel. Grav. 1127

[2] Sullivan B T and Israe] W 1980 Phys. Lett. 79A 371

[3] Lake K and Zannias T 1990 Phys. Rev. D 431798

[4] Kaminaga Y 1990 Class. Quantum Grov. 71135

[5] Landau L D and Lifshitz E M 1983 The Classical Theory of Fields (Oxford: Pergamon) section 25

[6] Dray T 1990 Class. Quantum Grav. 7 L131

[7] Barrabes C and Israel W 1990 Phys. Rev. D 431129 